\title{
Evaluation of PhonAge: An Adapted Smartphone Interface for Elderly People
}

\author{
Farah Arab, Yasir Malik, and Bessam Abdulrazak \\ University of Sherbrooke, Département d'informatique \\ 2500 boulevard de l'Université, Sherbrooke, Québec, Canada J1K 2R1 \\ \{farah.arab, yasir.malik, bessam.abdulrazak\}@Usherbrooke.ca
}

\begin{abstract}
Smartphones can play a significant role in maintaining decent Quality of Life for elderly people. Key factor to Smartphones usage success among elderly people depends on the accessibility of phone interface. In this paper, we present preliminary evaluation results of our PhonAge, an accessible and adaptable interface for Smartphones that is customized to the elderly profile. The results of the evaluation show adequacy of the interface to elderly needs. The evaluation feedbacks also helped in improving PhonAge interface design.
\end{abstract}

Keywords: Smartphone, Mobile Phone Interface, Accessibility, Evaluation, Aging.

\section{Introduction}

Smartphones can play a significant role in maintaining decent Quality of Life (QoL) for elderly people. It can promote independent living, active aging, and active socialization, while ensuring safety. The aging process is associated with progressive degradation of sensory, physical and/or cognitive abilities. The related disabilities deprive elderly people from independent living and maintaining social interaction [6], as a consequence they feel isolated and are transferred to special care facilities.

Recently, Smartphones have gained attention among elderly people and many of them are starting to use them for various purposes (e.g. as a reminder) [7]. Numerous services available on Smartphones can assist elderly people (e.g. navigation, interactive diary, activity reminder, speed dial, emergency service), however these solutions are not much welcomed in elderly community, either, because of accessibility issues, usability, elderly resistance and rejection toward technology, complex and tedious perception toward technology, etc. [3, 8]. At present, the mobile industry is targeting young and a tech-savvy population [5] and does not always take into account the special needs and expectations of the elderly population when designing services or phone devices. The main difficulties in adapting Smartphones for elderly people are limited or lack of personalization, complexity of mobile user interface (mUI) or a navigation menu, quantity of information arranged on screens, number of functions, language, etc. Consequently, mobile technology becomes more and more complex and elderly people are confronted with devices and services that are not adapted to their needs. We present in this paper a preliminary evaluation study 
that is conducted to test the usability of our solution named PhonAge [1], an accessible and adaptable solution for Smartphones that can host diverse useful services to elderly people. The results and feedback from this evaluation illustrates the adequacy of PhonAge to elderly needs, and helped in addressing the shortcomings of PhonAge interface.

The rest of the paper is organized as follow. In the next section, we present an overview of PhonAge, in section 3 we present the evaluation of PhonAge along with discussion on results in section 4 , and finally, in section 5 we conclude our work with direction on our future work.

\section{Overview of PhonAge}

PhonAge is an attempt to promote the use of smartphones among elderly people. In our experience while designing mHealthcare and social services on Smartphones for elderly people, we found that accessibility of phones is the major obstacle towards acceptance, usability and continuity of use [1]. To address such issues, we designed and evaluated PhonAge, an accessible and adaptable solution for Smartphones that is customized to the elderly profile and can host diverse services to assist them in their activity of daily living (ADL) [1]. PhonAge main interface/screen (Figure 1-a) is divided in three sections, namely (1) a spatiotemporal assistance section (Top section) to continuously provide elderly people with spatiotemporal contextual information (date, time and weather), (2) the Main activity and service area section to provide accessible phone feature and services to assist elderly and (3) an emergency section to ensure safety of elderly people while moving. PhonAge emergency button allows elderly people to alert someone in case of emergency (e.g. health problem, attack, wandering) according a predefined context-aware emergency protocol (based on the context-awareness framework presented in [2]). It also provides a configuration options that allow elderly people/caregiver/family to adapt Smartphones according to elderly needs, change the text and icon size, choose among the icons from gallery, change the order of icons, etc. (detailed presentation of PhonAge is available in [1]).

\section{Evaluation}

To study the usability and accessibility factors of PhonAge, we conducted a first stage evaluation with elderly people. The study aims at highlighting the compliance of PhonAge's mUI to the needs of elderly people and identifies usage difficulties. Following, we present evaluation method and preliminary results.

\subsection{Method and Data Collection}

To evaluate the mUI of PhonAge, we compiled a semi-structured interview supported by a questionnaire of about 45-60 minutes composed of 27 questions divided in three parts, and a direct observation of the PhonAge usage. The interview is designed to be 
conducted in person (by examiner) and the feedback and remarks to be recorded by the examiner. The questionnaire was pre-tested with one elderly people of age 65 . Following we describe three parts of questionnaire:

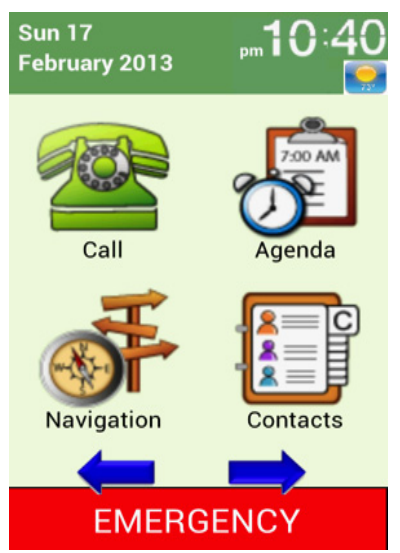

(a)

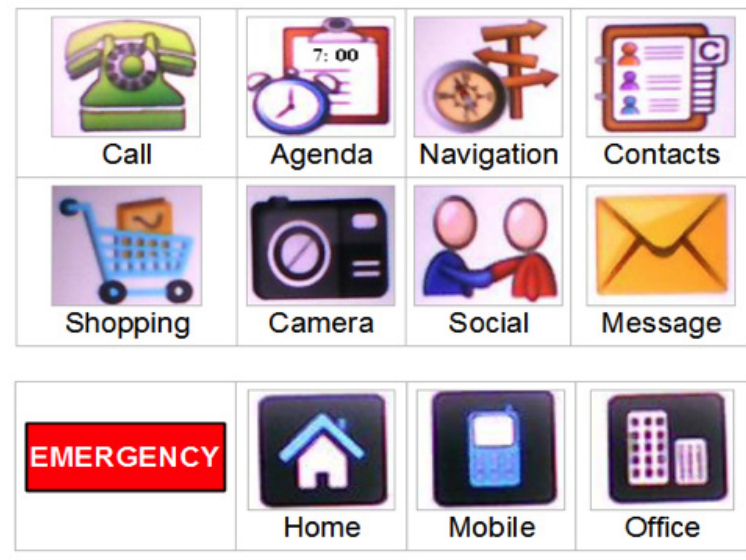

(b)

Fig. 1. PhonAge Main Screen (a) and Icon Presentation (b)

Part. 1. Gathering user information: In this part, we ask questions related to participants profile, such as age, education level, living environment (independent or nursing home), etc. The participants were asked to rate each item using a 5-point Likert scale.

Part. 2. Gathering details of outdoor ADLs: In this part, we ask questions about outdoor ADLs (i.e. purpose, frequency and organization of activities), kind of support for outdoor activities (e.g. mobility equipment device), use of mobile phone purpose (e.g. call, messages, reminder), frequency of mobile phone use, type of phones used (e.g. touch screen, dailpad).

Part. 3. Accessibility and Usability Test: In this part, we ask questions about understandability, usage, utility and satisfaction of the PhonAge interface.

- Icon Understandability Test is run to evaluate the meaning that PhonAge icons give (similar to [4]). First, participants are asked to give the meaning of the 12 icons proposed in PhonAge (figure 1-b). Second, we present the icons with their respective labels and ask the following question: "According to you which information do you consult if you touch the next icons". The order of the icons is modified to avoid inferring with any meaning.

- Use Test Method is run to evaluate the usability of PhonAge. The evaluation is designed to assess difficulties in use, utility of the navigation arrows provided (to switch between screens), and utility of functions (e.g. emergency, organization of icons/functionality on main screen). Participants are asked to navigate through phone functions and perform four defined tasks i.e. call, search contact address, search email and locate the camera function. 
Moreover, participants are asked to evaluate the PhonAge interface in terms of utility, facility of use and their preferences (i.e. interface structure, color of the wallpaper, services usefulness). We ask questions like "According to you, is it easy to? According to you, it is useful for outside activities to? According to you, the background is?"

\section{$4 \quad$ Results}

The results presented in this paper are those conducted with twenty elderly persons aged 60 to 84 (Mean=70 years, standard deviation=8, 2, 13 females and 7 males) who participated in this study. Following, we present the results.

\subsection{Activity of Daily Living}

In our study, most of the participants (19/20) live at home except one person lives in a retirement home. $11 / 20$ participants live with family, $5 / 20$ live in couple and $4 / 20$ by themselves. The results show that the main outings are grocery $(14 / 20)$, daily walk $(10 / 20)$, visit relatives (10/20), leisure (8/20), medical appointments (5/20), administrative activities (2/20) and volunteering (1/20). 10/20 participants prepare independently their outings, $8 / 20$ by writing a task list, $3 / 20$ plan their trips (e.g. bus schedules), and 3/20 assemble in advance documents for administrative activities. $7 / 20$ has been at least once victim of abuse outside. Half of the participants (10/20) also don't feel secure outside particularly because of the risk of street attacks or falls, vulnerability feelings and frailness. However, this insecure feeling decreases when someone accompanies them. Results show also that 11/20 participants have a mobile phone that they use either to call, to be contacted or as an event reminder (e.g. birthday or medical appointments). 3/20 participants also use the phone to send a message or take a photo, and 5/20 participants consult their directory (occasionally). Figure 2 illustrates the results of Use of mobile phones by elderly people.

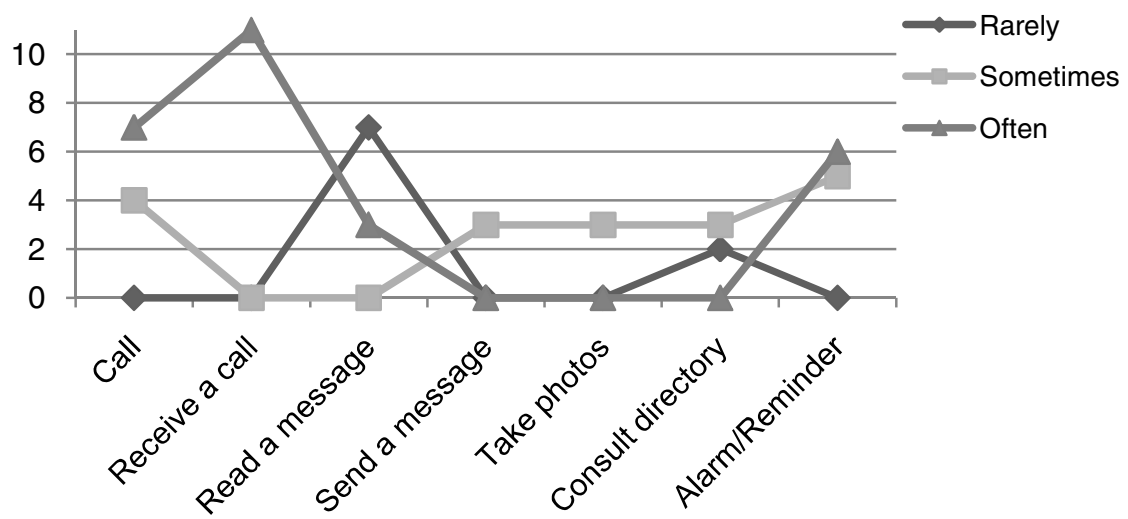

Fig. 2. Use of mobile phones by elderly people 


\subsection{Icons Understandability Test}

Evaluation of the icon understandability showed, that common icons (e.g. phone, emergency) were easily understandable (meaning and purpose) by all participants (20/20), even without a label (Figure 3 illustrates the results of the understandability of PhonAge icons). Labeling the icons helped participants to understand the navigation and social service icons. However, none was able to understand the following two icons: "shopping" for shopping list and "home phone number" on contact screen (Figure 5), 20 participants inferred that the shopping icon is for on-line shopping and not a shopping list. Similarly only one participant inferred that time shown on the agenda icon (7:00) (Figure 1) referred to an appointment at 7:00 AM. These results confirm the compliance of 9 icons to the associated functions. They also provided us with valuable feedback to modify the icons of shopping list, home and office phone. Taking into account our observation that elderly people pay attention to details/indications of icons, adding a sheet symbol to the "shopping" icon improved the understandability of the icon.

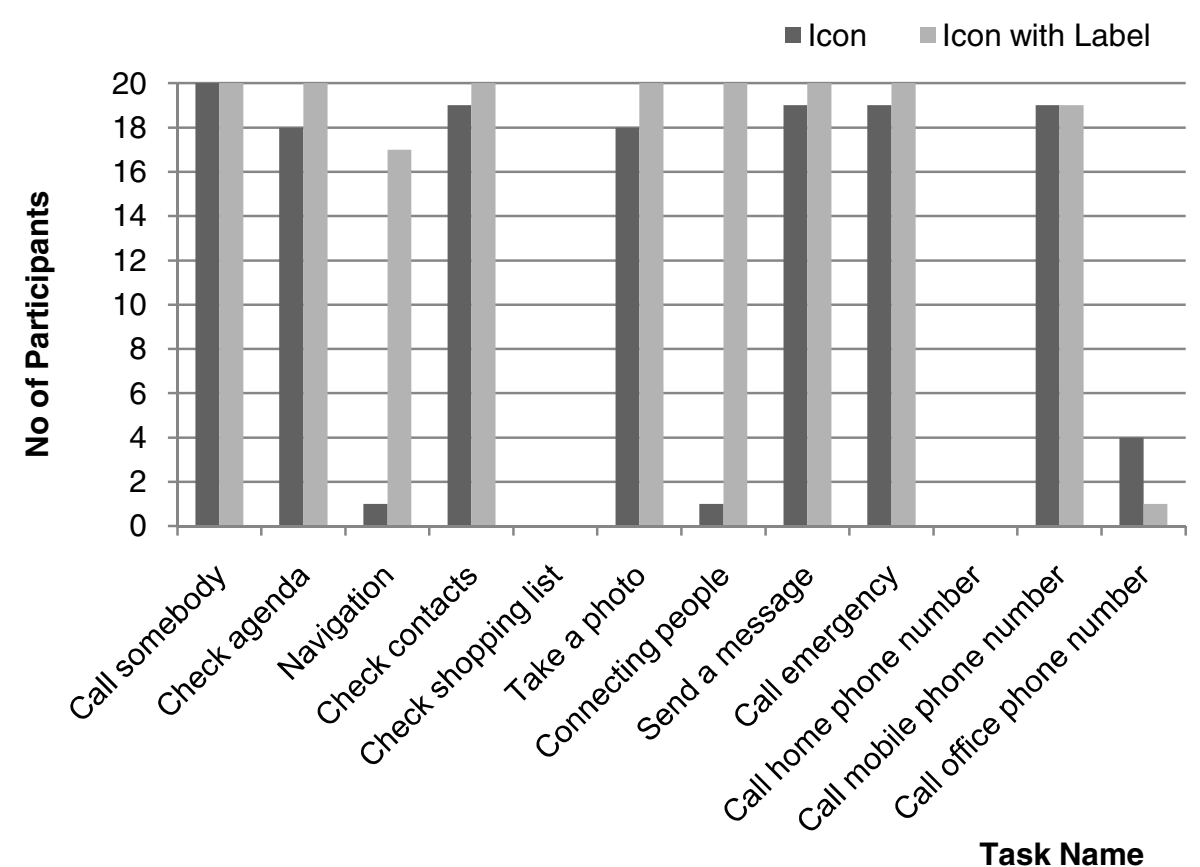

Fig. 3. Results of Icons Understandability Test

\subsection{Use Test}

Evaluation of PhonAge usage shows that the majority of participants (17/20) were able to easily execute the four proposed tasks (see section 2.1 Part3). Only 3 participants found these tasks difficult due to a visual impairment condition and their 
first time use of smartphones. During the first interaction with PhonAge, 17 participants used only the navigation arrows of the mUI to navigate through screens and icons selection (Figure 1-a). Only 3 participants used the touch scrolling, as they were already accustomed to it. After a demonstration time of the option (i.e. navigation arrows and touch scrolling), 17/20 participants preferred to keep both in the mUI. After this learning phase, participants found touch scrolling very intuitive as most of them said "we flip pages like in magazine, moving finger/hand form right to left." $16 / 20$ participants faced a difficulty to return to home screen (while navigation between screens). In the current version of PhonAge, returning to home screen is done always using the back button provided by the device. Since these participants were all novice Smartphone users, they were not aware of the presence of the button. One of the novice participants and the three who were already accustomed to touch screen devices had no problem returning to the home screen. After a learning phase of this option, the 20 participants were accustoming to the use.

The overall evaluation results shows that participants appreciated the clear color (e.g. green, yellow, blue) of the wallpaper and they appreciated the readability (16/20), visibility (20/20), pleasant aspects (18/20) and contrast (20/20) of the interface. Eighteen participants preferred the portrait display contrary to landscape display as they found it more classic.

The position of the icons and information on the main screen are also very much appreciated, for instance, all participants (20/20) liked the access to the spatiotemporal values and emergency button on all screens. Six participants also pointed, that one way to appropriate PhonAge is to personalize the label of the icons by adding the word "my" e.g. my contacts, my agenda, my shopping list rather than contacts, agenda, shopping.

\subsection{PhonAge Compliance to Preferences and Needs of Elderly People}

The use-test results confirmed the adequacy of PhonAge mobile interface design with elderly people perception. For most of the participants, the main purpose of using mobile phone is maintaining social relationships (calling and receiving calls, respectively $11 / 20$ and 15/20) and having safety services (contact somebody in case of emergency, 20/20). The most useful PhonAge's reported functions are: emergency (20/20), social service i.e. possibility to be connected with known and unknown people (17/20), and agenda to organize ADLs (15/20) (Figure 4 illustrates the results of PhonAge Service Usefulness).

In addition, participants found temporal information and its presentation very important; $16 / 20$ reported that date is important while $14 / 20$ reported time is important. However, 17/20 participants reported that weather information is not necessary to be available on all screens all the time. 15/20 participants reported that contact details is useful, they appreciated abstracted contact presentation (i.e. home, office and mobile contact information) (figure 5), and feel unnecessary to have detailed contact presentation (i.e. email, address, social contact). 


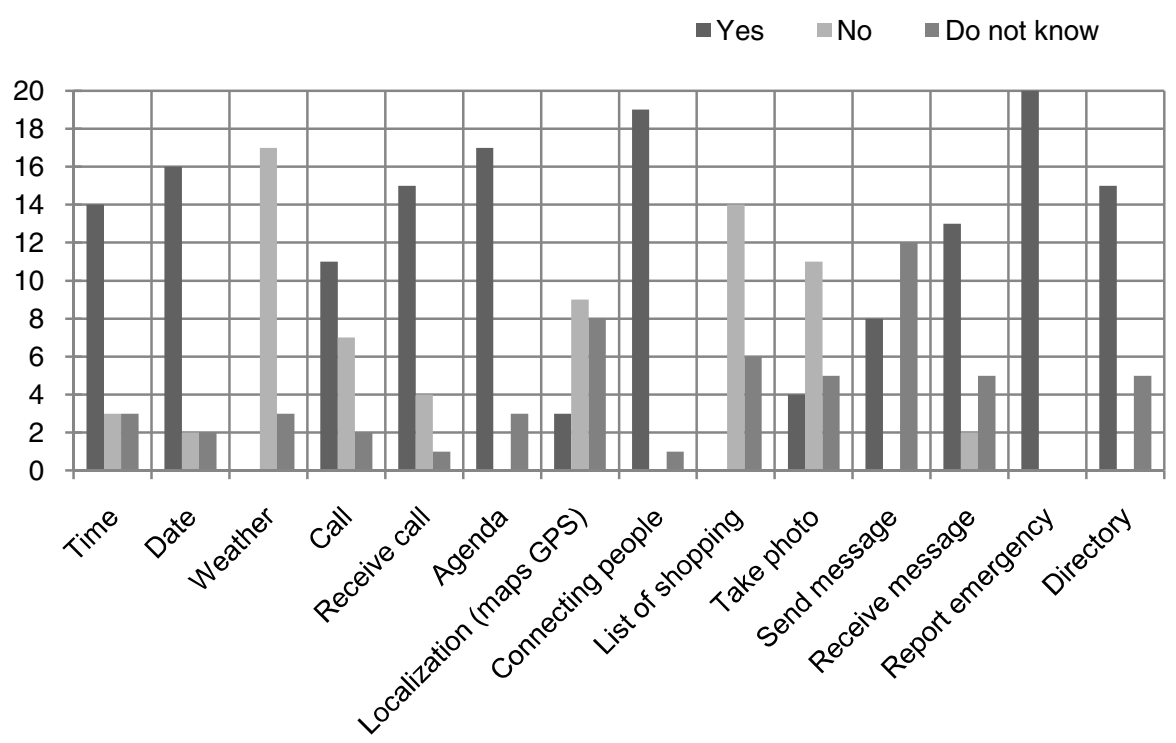

Fig. 4. PhonAge Service Usefulness

This feedback helped us to include the option of disabling detailed contact presentation in the configuration. Finally, only 3/20 participants (those already use smartphones) considered navigation service useful while other don't feel the need as their mobility is around their homes or they are accompanied with someone while going out. Only 4/20 participants find it interesting to take photos using smartphone camera, also all participants (20/20) appreciated the idea of displaying photos on the phone screen.

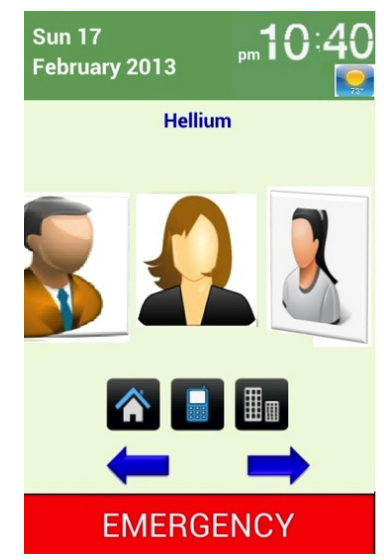

Fig. 5. PhonAge Contact Screen 


\section{$5 \quad$ Conclusion and Future Work}

We presented in this paper our first evaluation of PhonAge, a smartphone based solution that comply with elderly people profiles. This evaluation confirmed that PhonAge is quick to learn and its design meets the expectation and perception of elderly people. Moreover, the feedback received from the evaluation helped to improve and modify the icons so that elderly can have a better understanding of the function they use. The evaluation also highlighted the concern of participants regarding the data entry for contacts, daily activities, shopping list, etc. In future, we are working to develop and evaluate PhonAge' services in the context of Age Friendly cities that would assist elderly to remain independent and maintain social interaction. We encourage research community to use PhonAge, and welcome an opportunity to work together. Our goal is to build a system that promotes successful aging by use of PhonAge as a host that enables successful deployment of useful assistive services.

\section{References}

1. Abdulrazak, B., Malik, Y., Arab, F.: PhonAge: Adapted Smartphones to Assist Elderly in Age Friendly Cities. In: 11th International Conference on Smart Homes and Health Telematics, Singapore (2013)

2. Abdulrazak, B., Roy, P., Gouin-Vallerand, C., Belala, Y., Giroux, S.: Micro contextawareness for autonomic pervasive computing. International Journal of Business Data Communications and Networking 7(2), 48-68 (2011)

3. Arab, F., Pigot, H., Rabardel, P., Folcher, V., Rigaud, A.S., Mokhtari, M.: Age, memory and time: practices and support. Journal of Association for Advancement of Modelling and Simulation Techniques in Enterprises 71(3), 136-149 (2011)

4. Batu Salman, Y., Kim, Y.H., Cheng, H.I.: Senior friendly icon design for the mobile phone. In: 6th International Conference on Digital Content, Multimedia Technology and its Applications (IDC), Seoul, Korea, pp. 103-108 (2010)

5. Fisk, A.D., Rogers, W.A., Charness, N., Czaja, S.J., Sharit, J.: Designing for older adults: Principles and creative human factors approaches, vol. 2. CRC (2009)

6. Helal, A., Mokhtari, M., Abdulrazak, B.: The engineering handbook of smart technology for aging, disability, and independence. Wiley (2008)

7. Oksman, V.: Young People and Seniors in Finnish 'Mobile Information Society'. Journal of Interactive Media in Education (JIME) 2 (2006)

8. Ziefle, M., Bay, S.: How to overcome disorientation in mobile phone menus: a comparison of two different types of navigation aids. Human-Computer Interaction 21(4), 393-433 (2006) 